Revue pluridisciplinaire d'études médiévales

\title{
(Ré)utilisation de formes anciennes dans le décor sculpté de Beaulieu-sur-Dordogne
}

\section{Élise Haddad}

\section{(2) OpenEdition}

Édition électronique

URL : http://journals.openedition.org/questes/5370

DOI : 10.4000/questes. 5370

ISSN : 2109-9472

Éditeur

Les Amis de Questes

\section{Édition imprimée}

Date de publication : 28 février 2019

Pagination : 99-116

ISSN : 2102-7188

\section{Référence électronique}

Élise Haddad, « (Ré)utilisation de formes anciennes dans le décor sculpté de Beaulieu-sur-Dordogne », Questes [En ligne], 40 | 2019, mis en ligne le 19 avril 2019, consulté le 10 décembre 2020. URL : http:// journals.openedition.org/questes/5370 ; DOI : https://doi.org/10.4000/questes.5370

\section{(c) Association des amis de «Questes »}




\title{
(Ré)utilisation de formes anciennes dans le décor sculpté de Beaulieu-sur-Dordogne
}

\author{
Élise HADDAD \\ Gahom/Ahloma-CRH, EHESS, Paris, \\ I.I.S.E.R., Pune, Inde
}

L'église abbatiale romane de Beaulieu-sur-Dordogne est tout ce qui reste aujourd'hui du monastère bénédictin, qui a subi plusieurs destructions ${ }^{1}$. L'édifice roman fut commandité au début du XII siècle. Au moment où ce projet est porté, la communauté est d'obédience clunisienne. On date traditionnellement le décor sculpté autour de 1130, et en particulier le portail qui en est l'élément le plus important (images 1,2 et 3$)^{2}$. Néanmoins, l'iconographie de l'édifice ne se limite pas à ce portail. Or, si l'on s'intéresse au décor intérieur, on est frappé par la différence stylistique entre les chapiteaux, supposément contemporains de la construction, et les célèbres sculptures du portail. Plus encore, les trois linteaux sculptés qui surmontent trois portes intérieures semblent renvoyer à une esthétique et à une époque tout à fait différentes (image 5).

C'est cette disparité que nous voulons examiner ici. Nous chercherons à comprendre si le contraste esthétique résulte d'une obsolescence subie ou d'un usage conscient d'éléments désuets, dans le but de rendre visible un effet

\footnotetext{
${ }^{1}$ L'ensemble roman, construit au XII ${ }^{\mathrm{e}}$ siècle, a subi des dommages lors des guerres de religions. Il a été rasé et reconstruit par les mauristes. Puis, l'édifice mauriste lui-même a été démantelé, peu après la Révolution. Ces modifications successives rendent hasardeuses les hypothèses archéologiques précises sur la situation originelle de chaque décor déplacé.

${ }^{2}$ Cette date est établie par Émile Mâle, L'Art religieux du XII siècle en France, Paris, Armand Colin, 1922, p. 178.
} 
d'anachronisme. Et dans cette dernière éventualité, nous essaierons de comprendre quelle peut en être la signification.

À cet effet, nous comparerons plusieurs sculptures représentant des lions dans l'édifice de Beaulieu-sur-Dordogne, d'abord pour constater les différences de style frappantes entre ces éléments du même édifice. Puis, nous tenterons de comprendre ces différences non pas dans le seul contexte du style conçu comme domaine séparé, sans rapport avec les choix iconographiques, mais en combinaison avec les choix thématiques effectués pour chaque sculpture. Enfin, nous mettrons ces choix à la fois thématiques et stylistiques en lien avec la situation pour ainsi dire énonciative des sculpteurs. Il s'agira de les situer dans la chronologie de la production romane, par rapport aux références des générations précédentes, et par rapport aux innovations dont ils sont contemporains.

\section{Comparaison stylistique : l'exemple des lions}

Observons, dans un premier temps, les différences effectives qui apparaissent entre les différents objets sculptés. Afin de rendre cette comparaison plus saillante, nous extrayons un même motif, celui du lion, de ces ensembles sculptés à l'intérieur et à l'extérieur. En effet, la figure du lion est particulièrement présente à Beaulieu et elle est notamment traitée en plusieurs occurrences au portail, ainsi que sur au moins un des linteaux intérieurs.

\section{Les lions : redondance du thème, diversité des formes}

Notre corpus est constitué de cinq images. L'image 1 correspond au piédroit ouest du grand portail, c'est-à-dire du portail sud - on se reportera, pour la localisation, au plan fourni (image 6). On y voit le prophète Daniel en pied sous une arcade. Son nom est lisible à côté de son auréole. Il est entouré de sept lions, suivant en cela littéralement le texte biblique. La scène se réfère au célèbre épisode vétérotestamentaire de Daniel dans la fosse aux lions (Livre de Daniel, VI et XIV). 
Les images 2 et 3 présentent deux vues sur le bas du trumeau, au même portail, représentant également des lions. Cet élément est aujourd'hui très abîmé. Néanmoins, la juxtaposition d'un dessin de restitution(A), de la photographie (C), et d'un intermédiaire superposant le dessin et la photographie (B) permettent de rendre une certaine lisibilité à l'image. On y reconnait alors une composition de trois lions entrecroisés, dont les corps et les têtes circulent d'une face à l'autre de la colonne ${ }^{3}$. Cette composition au trumeau évoque des choix communs, possiblement un même atelier et sans doute un réseau de citations entre les édifices voisins de Moissac, Souillac et Beaulieu, où l'on observe aussi des entrecroisements de lions et d'animaux, tous réalisés dans le premier tiers du XII ${ }^{\mathrm{e}}$ siècle.

Enfin, sur l'image 5, sont superposés des linteaux situés à l'intérieur de l'église, respectivement au-dessus de la porte donnant sur le cloître et au-dessus des deux portes ouvrant sur des escaliers, dans les deux transepts. Les linteaux A et $\mathrm{B}$ donnent chacun à voir deux lions affrontés autour d'un élément central. Sur le linteau A, il s'agit d'un personnage dont les deux lions viennent lécher les mains. Sur le linteau B, c'est un végétal central dont les fauves broutent les feuilles. Le linteau $\mathrm{C}$ présente des bêtes considérablement plus féroces.

\section{Différences stylistiques}

Les images 1, 2 et 3, respectivement le piédroit et le trumeau du portail sud, font apparaître les différences internes, mais aussi les caractères essentiels attribuables à l'ensemble des lions. Ainsi, une certaine variabilité dans la profondeur du relief est observable, probablement due à la configuration du support. De même, diffèrent l'élongation des corps léonins ainsi que les choix de composition, en particulier la superposition et l'entrecroisement d'un corps

\footnotetext{
${ }^{3}$ Pour plus de détails, on pourra se reporter à Élise Haddad, «Common space or cleft places ? The example of Beaulieu, an architectural and figured space », dans Place and Space in the Medieval World, dir. Meg Boulton, Jane Hawkes et Heidi Stoner, London, Routledge/Taylor and Francis, 2017, p. 114-125.
} 
animal avec un autre. Il ne s'agit pas de déterminer la cause ou les motivations de ces différences : artisans distincts, degré de virtuosité, choix de proposer un écart pour le trumeau, etc. Disons du moins qu'elles ne produisent pas l'impression d'une différence dans la temporalité d'exécution. Ces éléments sont unanimement perçus comme faisant partie d'un même chantier de sculpture, de réalisation simultanée ou quasi-simultanée.

En revanche, le décalage est flagrant avec les linteaux de l'image 5. Regardons en particulier le linteau B, sur lequel la nature léonine des deux bêtes est incontestable car caractérisée par une crinière très fournie. Le relief y est considérablement plus aplati, cette fois non seulement dans la profondeur entre le fond de la sculpture et les objets sculptés, mais au sein même de chaque objet. En effet, chaque figure léonine semble constituée de plusieurs plans superposés, au sein desquels un détail supplémentaire se marque par l'incision d'une ligne. Plutôt que d'une réalisation en trois dimensions de même importance, il s'agit d'une sculpture dans laquelle deux dimensions sont traitées finement tandis qu'une troisième, la profondeur, permet le feuilletage en plusieurs plans distincts. Notons aussi sur le linteau C le caractère visiblement étrange, ostentatoire de l'archaïsme, notamment pour ce qui concerne la figure humaine centrale.

\section{Questions de datation}

Ces différences renvoient intuitivement les observateurs actuels à deux temporalités distinctes : les linteaux auraient été créés au $\mathrm{XI}^{\mathrm{e}}$, voire au $\mathrm{X}^{\mathrm{e}}$ siècle ${ }^{4}$. Ils produisent un effet d'anachronisme. On sait la fragilité d'hypothèses

\footnotetext{
${ }^{4}$ Ces linteaux n'ont jamais fait l'objet d'une étude monographique publiée. Pour une synthèse relativement récente, voir Anne-Marie Pêcheur et Évelyne Proust, «Beaulieu-sur-Dordogne, abbatiale Saint-Pierre », dans Monuments de Corrèze. Congrès archéologique de France (163 session, 2005), Corrèze, Société française d'archéologie, 2007, p. 83-103.
} 
chronologiques appuyées exclusivement sur le style ${ }^{5}$. Un tel décalage peut aussi bien refléter la survie d'un savoir-faire passé de mode, que l'affirmation volontaire d'un style «antique», ou encore une date de production effectivement antérieure. Des indices archéologiques pourraient plaider en faveur de la réutilisation, c'est-à-dire la réinsertion d'éléments antérieurs dans un nouvel ensemble. Mais dans le cas des linteaux de Beaulieu, ces éléments restent fragiles. Le caractère ostentatoirement archaïsant, étrange même, du linteau $\mathrm{C}$, combiné dans ce même linteau avec une facture tout à fait habile des motifs végétaux, pourrait faire pencher vers un anachronisme volontaire. Il pourrait encore s'agir d'une reprise avec modification de l'élément sculpté. Plutôt que de nous attarder sur la raison de ce style archaïsant, nous prendrons ici en compte la signification et les effets produits par ce linteau, une fois ce dernier inséré dans le contexte de l'église.

Que ces linteaux aient fait l'objet d'une récupération et d'une réutilisation à partir d'un édifice antérieur, ou qu'ils constituent l'utilisation volontaire d'un style archaïsant, nous nous attachons ici à l'effet sémantique construit par leur agencement et leur différence avec l'ensemble sculpté principal.

En effet, si ces sculptures sont réutilisées sans prise en compte intentionnelle de l'effet d'anachronisme que produit leur juxtaposition au style sophistiqué du portail, alors elles relèvent de l'obsolète au sens qui a été défini dans le cadre de la présente publication : toujours en place, quoique visiblement datés, ces éléments fonctionneraient pour ainsi dire malgré leur défaut de pertinence stylistique. En revanche, si cet effet de décalage chronologicostylistique est voulu, ou simplement réinvesti dans l'agencement et la production du sens, on pourra, toujours selon la grille d'interprétation de ce volume, parler de désuet: le contraste et la rupture introduits par l'anachronisme sont

\footnotetext{
${ }^{5}$ Pour une célèbre mise au point sur ces questions bien connues, voir Jean Wirth, La Datation de la sculpture médiévale, Genève, Droz, 2004, p. 103-108.
} 
signifiants, et la tonalité archaïque participe à la construction d'une fonction nouvelle, d'une dimension supplémentaire dans la production du sens.

\section{Distinction thématique : Éden primordial et épreuves finales}

Il nous faut d'abord observer et comparer les thématiques abordées par le groupe des linteaux d'une part, et par le groupe du portail d'autre part. Là encore, par souci de continuité, nous nous attachons à la figure du lion.

\section{Les lions aux linteaux : ambiguïté du danger}

Dans chacun des trois linteaux visibles sur l'image 5 (A, B et C), deux fauves sont représentés. Les bêtes des linteaux A et B sont symétriques et placides. Leur attitude peut surprendre. Certains ont cru reconnaître dans le linteau A une représentation de Daniel entre les lions ${ }^{6}$. Néanmoins, certains éléments nous poussent à adopter une position plus prudente. D'une part, seule l'attribution des lions permet d'identifier le prophète. Or, justement, la comparaison avec le linteau B est sans appel : lorsque les sculpteurs des linteaux souhaitent faire reconnaître des lions, ils donnent à voir des marques fortes de l'espèce. La crinière joue alors un rôle prépondérant. Au linteau A, il semble qu'on ait voulu en rester à des marques plus générales, comme il est commun dans la sculpture romane ${ }^{7}$ : ces deux bêtes sont des quadrupèdes réduits à leurs stéréotypes. Le linteau A constitue donc une mise en image de l'innocuité des bêtes, dans une relation pacifique mais hiérarchique entre homme et animal.

Dans le linteau B, la scène est encore plus surprenante : les deux lions, clairement identifiés dans leur espèce, broutent une plante décorative, dont

\footnotetext{
${ }^{6}$ Il est identifié ainsi et classé dans la catégorie des occurrences certaines de Daniel dans la fosse aux lions par Juan Antonio Olañeta, dans le catalogue de sa thèse : La Iconografía de Daniel en el foso de los leones. Las transformaciones de una imagen en la escultura del Occidente europeo (ss. XI-XII), thèse en préparation sous la direction de Carles Mancho à l'université de Barcelone.

${ }^{7}$ Jérôme Baschet, Jean-Claude Bonne et Pierre-Olivier Dittmar, Le Monde roman par-delà le bien et le mal. Une iconographie du lieu sacré, Paris, Arkhê, 2012, p. 99-107.
} 
l'espèce végétale reste méconnaissable. Ces lions sont herbivores. À ce point de l'observation, il faut faire intervenir une référence biblique afin d'éclairer le thème représenté. Il s'agit de Genèse, I, 29-30 :

29 dixitque Deus ecce dedi vobis omnem herbam adferentem semen super terram et universa ligna quae habent in semet ipsis sementem generis sui ut sint vobis in escam

30 et cunctis animantibus terrae omnique volucri caeli et universis quae moventur in terra et in quibus est anima vivens ut habeant ad vescendum et factum est ita $^{8}$.

Les lions du linteau B sont donc par excellence des bêtes inoffensives, en ce qu'elles n'ont pas à chasser d'autres animaux pour s'en nourrir. Leur affinité avec le règne végétal est soulignée par la végétalisation de leurs corps : on peut notamment observer à cet égard les extrémités des queues. Les linteaux A et B forment un ensemble, faisant allusion à une forme d'Éden originel dans lequel toutes les bêtes pouvaient vivre en paix, sans danger pour les unes ni pour les autres. Cet Éden est donné en modèle au monastère.

Comment comprendre dès lors le linteau $\mathrm{C}$, qui détonne par sa composition et par la relation homme/animal qu'il représente ? La symétrie y est en effet désaxée : l'homme, élément central, ne se situe pas au centre, mais légèrement à droite de la pointe du triangle formée par le gable. De fait, c'est l'équilibre de l'ensemble qui est menacé.

À droite, une bête visiblement féroce, munie de crocs et de griffes, est tenue en respect, mais ce n'est plus spontanément qu'elle se rend; il y faut une laisse. Au centre, l'homme est muni d'une arme pour affronter les animaux qui l'entourent. La coopération volontaire n'est plus de mise. Enfin, du côté gauche,

\footnotetext{
${ }^{8}$ «Et Dieu dit: voici que je vous ai donné toute herbe portant graine sur la terre, et l'ensemble des plantes qui portent en elles-mêmes les graines de leur espèce, pour qu'elles soient votre nourriture - à tous les êtres animés de la terre, et à tous les oiseaux du ciel, et à l'ensemble de ce qui bouge sur terre et en qui est une âme vivante, pour qu'ils en fassent leur pâture. Et ainsi fut fait » (nous traduisons, à partir du texte de la Vulgate disponible en ligne sur Bible Gateway : www.biblegateway.com).
} 
c'est bien pire: une bête aux longues dents tient dans sa gueule un petit herbivore, un mouton renversé, tête en bas. Il s'agit peut-être d'un loup ${ }^{9}$, mais aucune caractéristique spécifique ne permet de l'affirmer. Quoi qu'il en soit, toute affinité entre carnivores et herbivores est ici écartée. Les deux catégories sont bien distinctes : l'ovin arbore des pattes végétalisées en analogie graphique avec l'élément végétal qui court en bas de l'image, au centre du linteau; les carnivores, plus du tout. Le règne animal a été divisé en deux avec l'homme pour arbitre, armé pour dominer cet ensemble brisé et lui imposer un ordre.

D'un point de vue purement thématique, cet ensemble de trois linteaux s'articule donc autour de la question de la bête et de sa possible domesticité ou dangerosité. L'alternative est posée : innocuité originelle et idéale, ou danger réel et actuel contre lequel l'être humain doit se prémunir.

\section{Les lions au portail : ambivalence ${ }^{10}$ de l'épreuve}

Au grand portail sud, la situation est différente. Nous avons observé deux groupes de lions : ceux qui entourent Daniel et ceux du trumeau. Sans entrer dans le détail de l'iconographie, tous ces lions ont en commun de soutenir, au sens de soutènement architectural, soit le tympan, soit d'autres lions juchés sur les premiers, ou encore Daniel lui-même.

Autour de Daniel, ce rôle est mis en évidence de plusieurs manières. L'un des lions sert de siège au prophète, c'est-à-dire de soutènement physique. À droite, quatre lions viennent former une pyramide, image vivante de la colonne. En symétrie, les deux lions de gauche, également superposés à la verticale l'un de l'autre, jouxtent une colonne, qui vient couper la scène sans nécessité architecturale. Cette colonne, apposée au corps des deux lions, mise en symétrie avec la colonne léonine de droite, désigne directement le rôle qui est attribué à nos bêtes.

\footnotetext{
${ }^{9}$ La sculpture s'appuierait alors sur un épisode du Livre d'Isaïe, LXV, 25.

10 Jean-Claude Bonne, «Entre ambiguïté et ambivalence (problématique de la sculpture romane) », La Part de l'œil, 8, 1992, p. 146-164.
} 
Quant aux lions du trumeau, il n'est pas besoin d'insister sur leur rôle de soutènement : leur enchevêtrement acrobatique, les appuis des uns sur les autres, leur agencement avec les colonnettes, tout concourt à renforcer notre hypothèse. Pour s'en convaincre un peu plus, on observera l'image 4, une vue générale du trumeau : les lions servent de soubassement à un autre porteur, humain cette fois, au corps élancé et à la nuque brisée sous le poids du linteau, doté de larges mains ouvertes sous la pierre.

Néanmoins, une autre propriété, concomitante mais concurrente à cette puissance d'appui, mérite que l'on s'y attache. Sur l'image 3, on voit que l'un des lions, tout en faisant partie de l'enchevêtrement acrobatique, vient mordre la jambe d'un atlante humain. Sans cesser d'être porteur de l'édifice, ce lion menace simultanément son soutien. Il porte et sape à la fois. Il assure l'équilibre et le met en danger. Il annonce ou dénonce l'ambivalence inhérente à la position du lion.

\section{Deux discours adressés à deux publics}

Ces deux discours bien distincts sont situés dans des zones de l'édifice déterminées, comme on peut l'observer sur le plan de l'église (image 6). Le portail sud est un ensemble compact, situé au flanc de la nef, visible sur le plan à la hauteur de la deuxième travée. Il est situé du côté de la ville, sur la place du marché. Il est visible par les laïcs, depuis l'extérieur et lorsqu'ils pénètrent dans l'édifice par l'entrée principale qui leur est dédiée. Car l'église de Beaulieu est à la fois paroissiale et abbatiale, et marquée par la cohabitation de deux catégories de fidèles.

Les trois linteaux semblent se situer plutôt dans l'espace dédié aux moines. Pour deux d'entre eux, ce positionnement est univoque : les linteaux A et $\mathrm{B}$ se situent du côté nord, jouxtant le monastère, respectivement dans la nef et dans le transept. Le linteau A surmontait originellement la porte menant au cloître. Le transept nord, dans lequel se trouve le linteau B, communique encore 
aujourd'hui avec l'ancienne salle capitulaire et aurait autrefois permis de rejoindre les bâtiments monacaux au premier étage. Ces deux éléments si complémentaires se situent donc résolument dans l'espace de la clôture.

En réalité, il est possible de délimiter plus précisément les espaces au sein de l'édifice. Les couleurs visibles sur l'image 6 rendent compte d'une répartition iconographique : en vert, les deux premières travées de la nef sont décorées de motifs simples et exclusivement végétaux. Le reste de l'édifice est en revanche peuplé de figures animales, voire humaines, non seulement sur les trois linteaux que nous connaissons mais aussi sur les chapiteaux, représentés par de petits cercles. Précisons que le constat stylistique dont nous sommes partis se superpose à ce découpage : nous avons jusqu'ici concentré notre regard et notre attention sur les lions des linteaux, mais les chapiteaux de l'espace coloré en jaune portent les mêmes caractéristiques d'archaïsme, et parfois avec une ostentation comparable à celle que nous avons constatée au linteau C. Enfin, nous disposons de quelques indices archéologiques dans les pierres des murs qui sont encore en place, ce qui permet de formuler une hypothèse quant à la localisation de la barrière physique matérialisant la clôture monacale ${ }^{11}$.

D'après nos hypothèses iconographiques et archéologiques, le linteau $\mathrm{C}$, différent des deux autres en termes de thématique, se situe toujours dans l'espace des moines, mais dans le transept sud, sur le chemin de la porte qui mène vers la ville. Il s'adresse donc aux moines au moment où ils vont sortir dans le monde et se mêler aux laïcs.

Ainsi, il apparaît bien que les différences stylistiques à coloration chronologique du groupe des linteaux (et, plus généralement, d'une certaine partie de l'intérieur) avec celui du portail correspondent à une distinction de

\footnotetext{
${ }^{11}$ Pour une argumentation précise, voir Élise Haddad, Le bien à l'épreuve du mal. Autour du tympan de Beaulieu-sur-Dordogne, eschatologie conflictuelle dans l'image médiévale, et son fonctionnement dans l'ontologie analogique, perspectives diachroniques (titre provisoire), thèse de doctorat, EHESS, Paris, 2019, chap. 2, F, p. 180 et suivantes.
} 
signification. Elles reflètent des thématiques différentes, des valeurs et des problématiques différentes de la figure du lion, des destinataires différents - en somme, deux discours irréductibles l'un à l'autre. En cela, le procédé stylistique mis en jeu relève nettement du désuet, c'est-à-dire de l'usage volontaire d'un caractère passéiste pour produire un contraste avec le style le plus virtuose et nouveau.

Il nous reste néanmoins à expliquer la pertinence du recours spécifique à la tonalité chronologique pour marquer cette différence. On comprend bien en quoi le décalage introduit par l'anachronisme correspond effectivement à un décrochage discursif. Mais parmi tous les marqueurs possibles, pourquoi avoir choisi en particulier celui de la coloration chronologique?

\section{Distinction chronologique : une parole ancestrale}

Dans un premier cas, sur les linteaux (image 5), nous avons rencontré des figures de bêtes soit inoffensives, soit dangereuses. La question de la relation à la bête est posée, ainsi éventuellement que celle du processus de domestication. Dans un second cas, au portail (images 1, 2, 3 et 4), les lions sont entièrement intégrés et nécessaires à l'édifice, et il n'est pas question de les tenir à distance ; la domestication a eu lieu. Néanmoins, la capacité de nuire demeure et se combine à ce nouveau rôle.

\section{Temporalité des événements}

Les représentations portées sur les linteaux, à l'intérieur de l'édifice, s'articulent avec un passage de la Genèse. Cette référence est explicite au linteau B, dans la figure des lions broutant. Elle est aussi une clef de compréhension directe du linteau A aux deux bêtes inoffensives. Il s'agit donc d'un discours portant sur les origines mythiques : une parole au passé. Certes, l'état originel idéalisé est réactualisé : placé dans l'espace des moines, il leur est proposé comme modèle. Ces représentations assimilent le monastère à l'Éden 
réinstauré, ou au paradis à venir. L'harmonie entre êtres animaux et humains y est aussi une analogie de l'harmonie intérieure du moine et des forces qui l'habitent. Cet état se joue hors du temps. Néanmoins, la référence à un état passé, et pour l'essentiel perdu, est bien présente. L'éloignement temporel de ce passé est de fait rendu visible par l'utilisation d'un style ancien, déjà obsolète ou désuet au XII ${ }^{\mathrm{e}}$ siècle.

De plus, le linteau C resitue l'ensemble précisément dans une dimension temporelle, celle d'un processus : la phase de domptage de bêtes ensauvagées par la Chute de l'Homme. L'ensemble de ce discours porte sur un temps révolu - celui de l'Éden - ou sur un temps dont l'accomplissement est déjà bien engagé : la domestication des bêtes. Le choix de mettre en ouvre ou de conserver des marqueurs désuets pour ces représentations est une manière de faire résonner leur contenu spécifique dans sa valeur temporelle - celle du passé.

$\mathrm{Au}$ portail en revanche, le contexte est tout autre. L'ensemble s'articule autour d'un grand tympan eschatologique : la dimension temporelle principale est le futur. En réalité, la thématique développée dans l'ensemble du portail est celle de l'épreuve ${ }^{12}$, qui mène du présent terrestre - tentations, difficultés de la vie - au futur eschatologique - épreuve ultime donnant accès à l'éternité. Le lion s'insère dans ce schéma comme une force terrestre, désignée comme essentielle par sa place architectonique, mais tout de même menaçante. La force du corps animal est utile, mais elle peut se retourner en morsure, épreuve à surmonter pour pouvoir rester un pilier de l'Église. Sans élaborer plus avant cet ensemble sémantique complexe, nous pouvons néanmoins le situer sur un axe temporel : les représentations du portail sud valent dans un temps qui s'étend du présent au futur. En cela, le style virtuose déroulé au tympan sert à désigner, dans une forme du tout dernier cri, les évènements du tout dernier moment.

La coloration désuète que nous avons repérée, en contraste avec le modernisme du grand portail sud, désigne aussi dans les deux ensembles de

\footnotetext{
${ }^{12}$ Pour une démonstration approfondie, ibid., chap. 1-2.
} 
représentations deux objets temporels distincts : des origines au présent dans un cas, et du présent aux fins dernières dans l'autre.

\section{Temporalité de l'énonciation}

Hasardons enfin une dernière hypothèse à propos de la temporalité de ces deux ensembles visuels. Il nous semble que l'énonciation de ces deux discours relève de générations successives de parole.

Revenons sur les moments de production de ces discours : l'espace de création roman a donné lieu à plusieurs phases discursives sur le rapport entre animaux et humains, comme analogues du rapport spirituel intérieur à l'homme $^{13}$. Les sculptures des linteaux produisent précisément un discours plus positif quant à la possibilité d'un rapport pacifique avec l'animal, d'une relation de coopération consentie, et font partie d'une première génération d'images ${ }^{14}$. L'iconographie du portail, en revanche, fait un usage ambivalent et dans certains cas explicitement négatif de la bête, non seulement par la morsure du lion au bas du trumeau, mais par tout un réseau de bêtes représentant les plaies apocalyptiques, et de monstres dentus en pleine entreprise de dévoration. La menace ne se résout pas par une collaboration avec l'animal mais constitue une tension permanente, un rapport de force et de résistance à maintenir. Au trumeau comme dans la majorité des représentations au portail, la domination sur la bête est une action précaire et toujours à renouveler contre la volonté de l'animal.

Notons que notre petit corpus léonin, incluant Daniel, nous permet ici une dernière observation : au sein même du portail, le piédroit ouest, celui de Daniel, est le lieu de l'Ancien Testament, du passé. Or c'est précisément le seul espace

\footnotetext{
${ }^{13}$ La possibilité d'interpréter les incarnations animales comme des représentations de la part pulsionnelle de l'humanité n'est qu'en formation au XII siècle : voir Pierre-Olivier Dittmar, Naissance de la bestialité. Une anthropologie du rapport homme-animal dans les années 1300, thèse de doctorat sous la direction de Jean-Claude Schmitt, EHESS, 2010, chap. III, et plus spécifiquement p. 344-359.

${ }^{14}$ Jérôme Baschet, Jean-Claude Bonne et Pierre-Olivier Dittmar, Le Monde roman par-delà le bien et le mal, op. cit. Sur cette évolution spécifiquement, voir p. 113-124.
} 
dans lequel les bêtes sont inoffensives, sans équivoque. Voici un indice, s'il en fallait, du fait que l'assignation d'un discours au passé et d'un autre au présent est un processus assumé dans l'édifice de Beaulieu, et non le résultat d'un réemploi pragmatique et irréfléchi.

La différence stylistique à tonalité chronologique que nous avons observée dès le début entre les lions de l'intérieur et de l'extérieur de l'édifice correspond bien à la constitution de deux discours distincts. Il s'agit d'une mise en scène volontaire du caractère désuet d'une esthétique sculptée, servant à marquer le décrochage entre un discours et un autre, répartis chacun dans un espace distinct de l'édifice.

L'un de ces discours, à l'intérieur de l'édifice, se lit essentiellement comme un discours au passé. Il porte sur un évènement déjà révolu, celui de la Chute, et sur un processus largement accompli, celui de la domestication. L'autre discours, au grand portail, s'articule à un contexte eschatologique. Il s'adresse à l'ensemble du peuple des croyants dans une temporalité qui s'échelonne du présent au futur. Le choix d'une esthétique archaïsante pour mettre en image le premier discours n'est donc pas un marqueur arbitraire : il s'agit de tirer parti, explicitement, de la désuétude engendrée par la forme.

Enfin, dans un jeu de double assignation des discours, par leurs contenus et par les conditions de leur énonciation, les représentations des linteaux se rattachent également à une énonciation au passé, en ce qu'elles appartiennent à une tradition iconographique attestée dans les générations précédentes mais en phase d'obsolescence; l'analogisme du portail correspond pour sa part à un discours conquérant, matérialisé dans une esthétique inventive et novatrice.

L'exemple de Beaulieu et de son décor sculpté incite à observer de plus près les phénomènes d'archaïsme et les effets de désuétude présents dans de nombreux ensembles romans. Qu'ils soient dus ou non à des réutilisations, les fabricants d'images médiévaux les mobilisent de manière maîtrisée, et les dotent 
de sens riches et multiples. Il vaut donc la peine d'en examiner les ressorts efficaces de signification. 


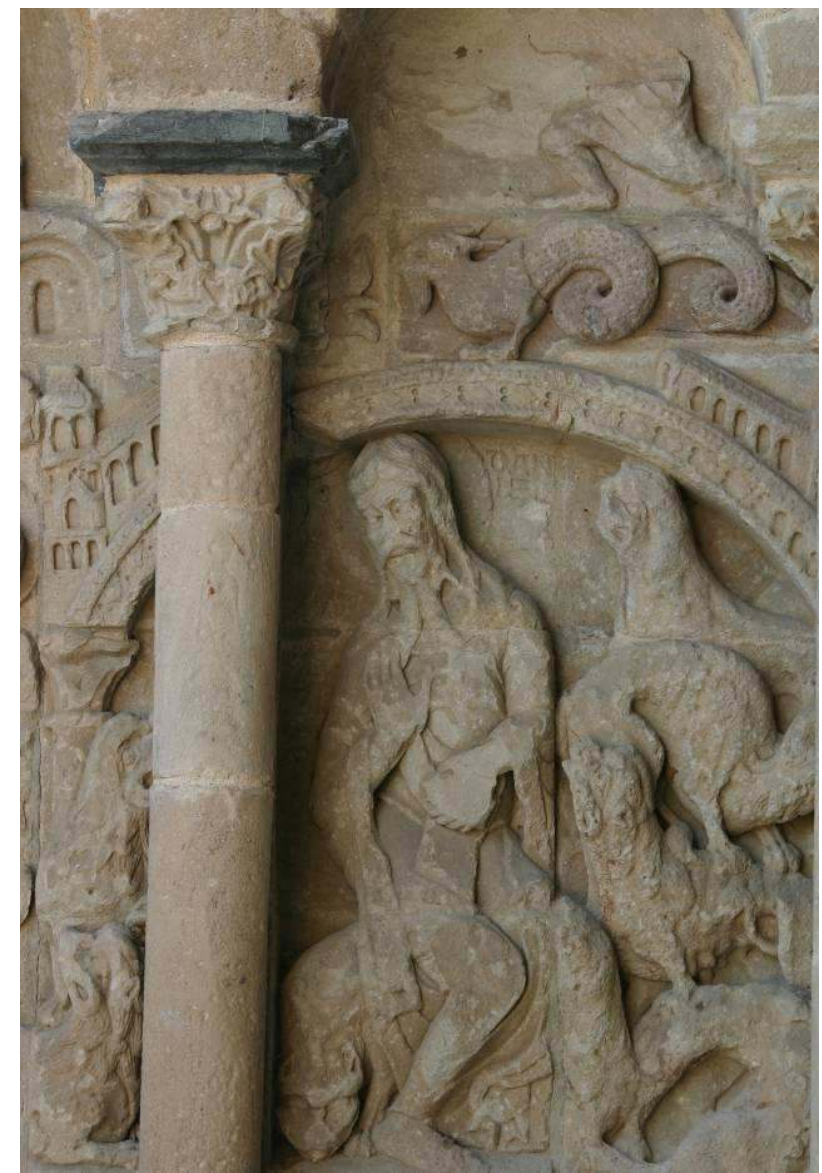

Image 1 : Église de Beaulieu-sur-Dordogne, portail sud, piédroit ouest : Daniel dans la fosse aux lions.

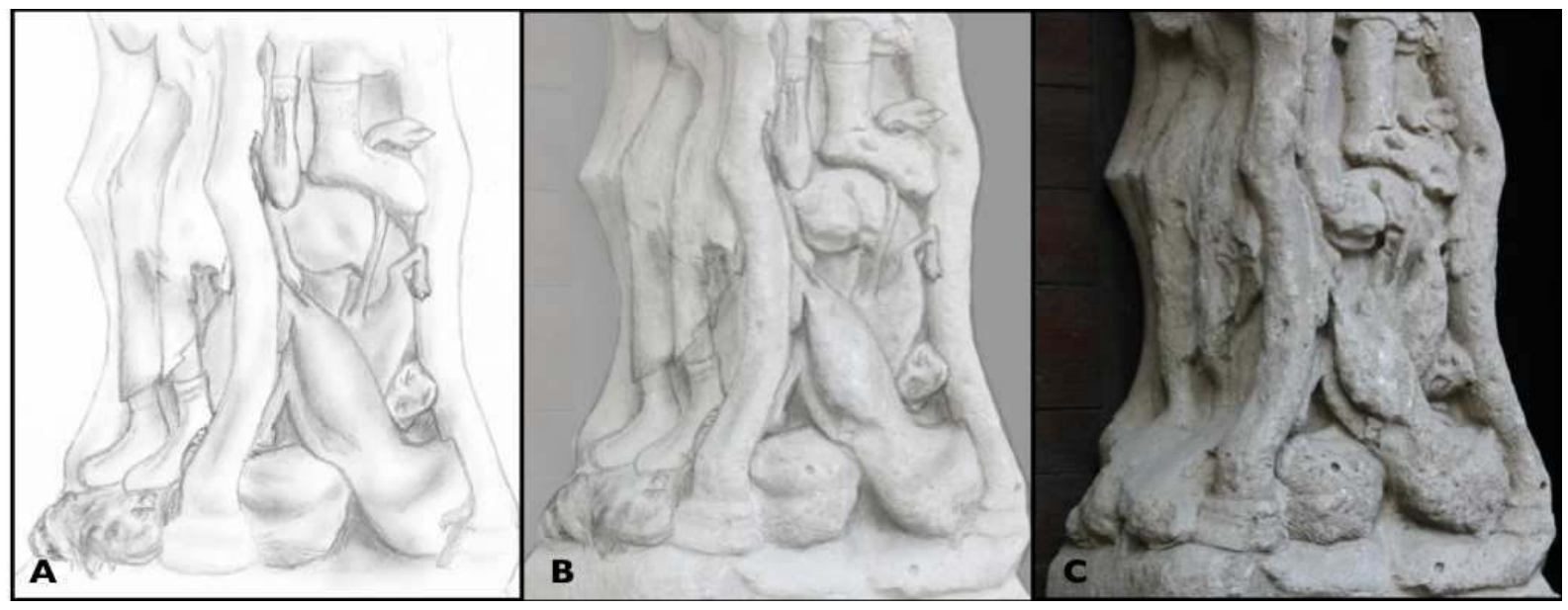

Image 2 : Église de Beaulieu-sur-Dordogne, portail sud, trumeau vu depuis le sud-ouest : pyramide de lions. A : dessin ; B : dessin surimposé à la photographie ; C : photographie. 


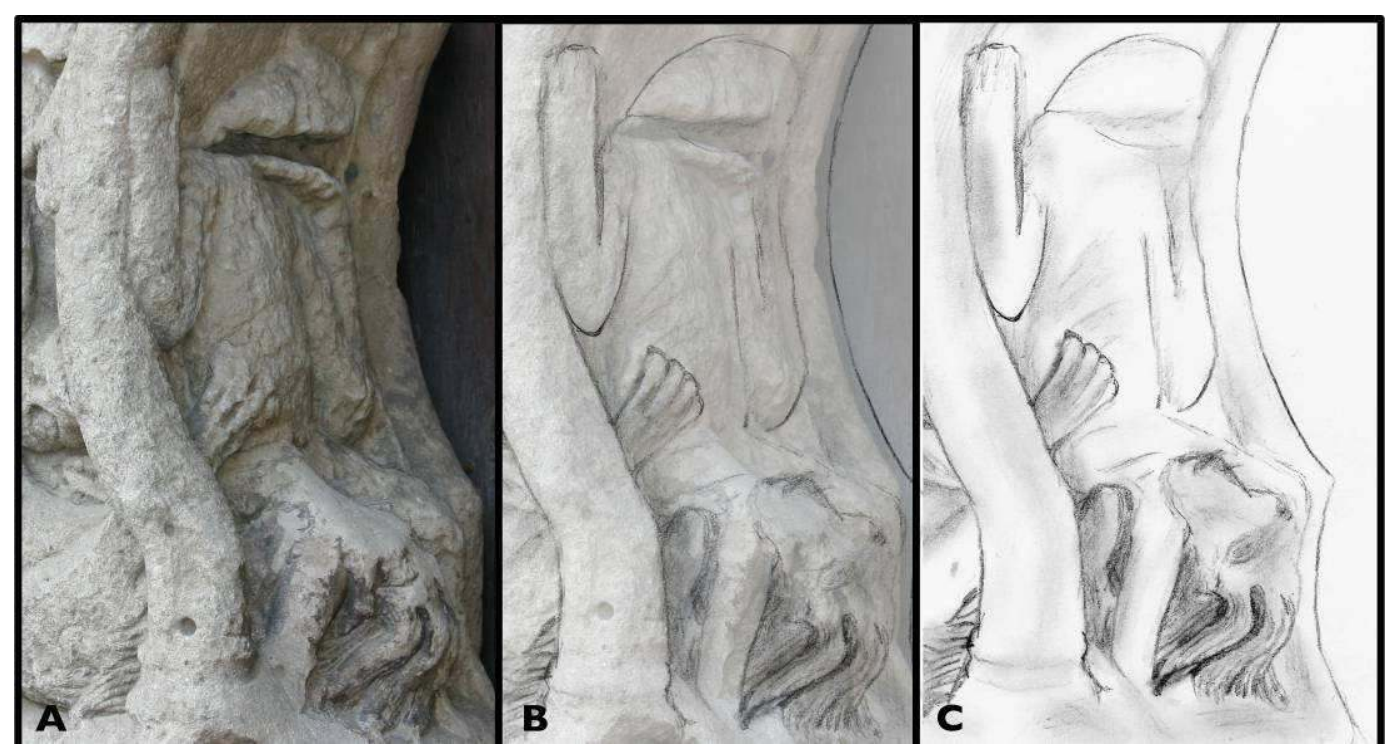

Image 3 : Église de Beaulieu-sur-Dordogne, portail sud, trumeau vu depuis le sud-est : lion mordant un personnage. $A$ : photographie ; $B$ : dessin surimposé à la photographie ; $\mathrm{C}$ : dessin.

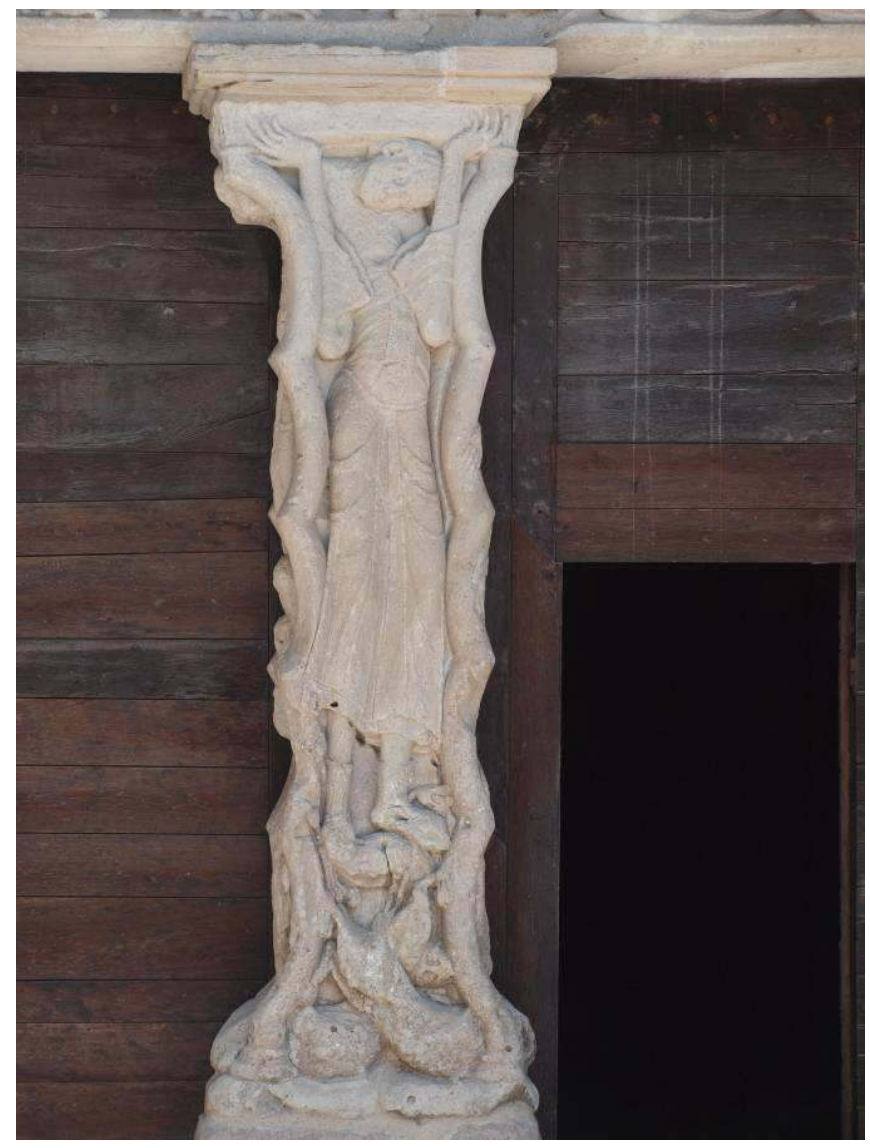

Image 4 : Église de Beaulieu-sur-Dordogne, portail sud, trumeau vu depuis le sud : porteurs humains et lions. 


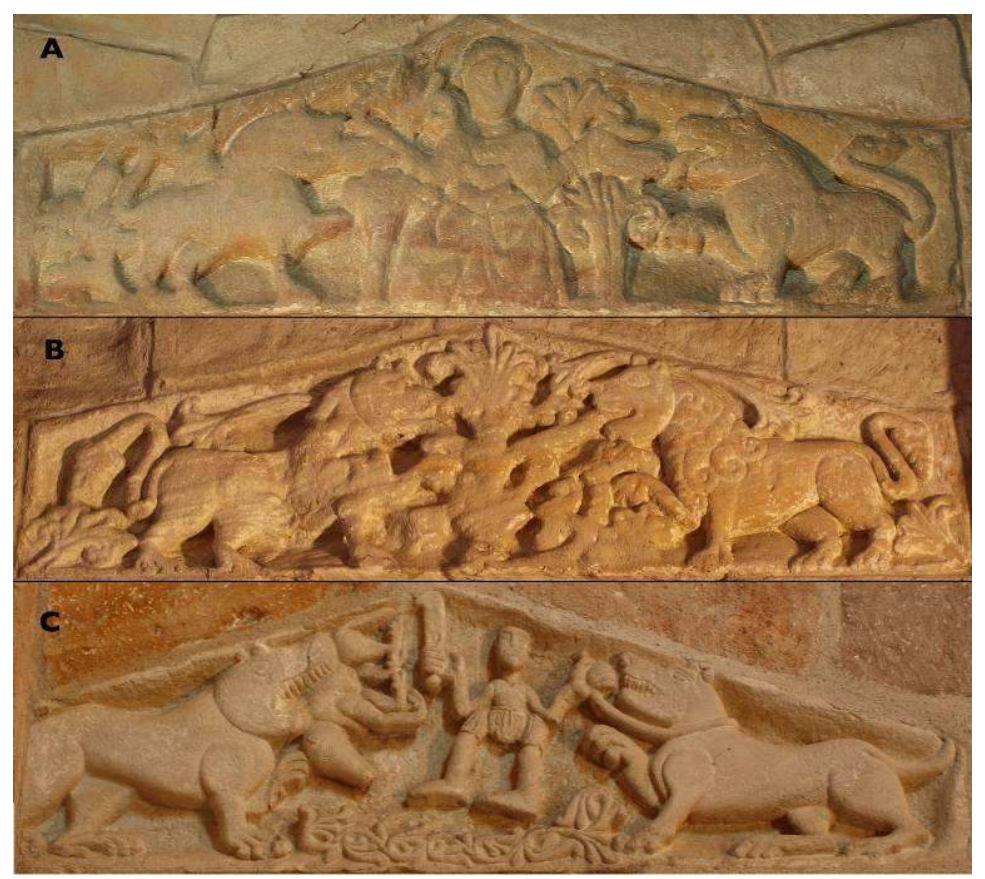

Image 5 : Église de Beaulieu-sur-Dordogne,

linteaux intérieurs. A : bas côté nord, un homme entre deux lions ; B : transept nord, deux lions broutant un végétal ; $C$ : transept sud : une bête croquant un mouton et une autre tenue en laisse par un homme.

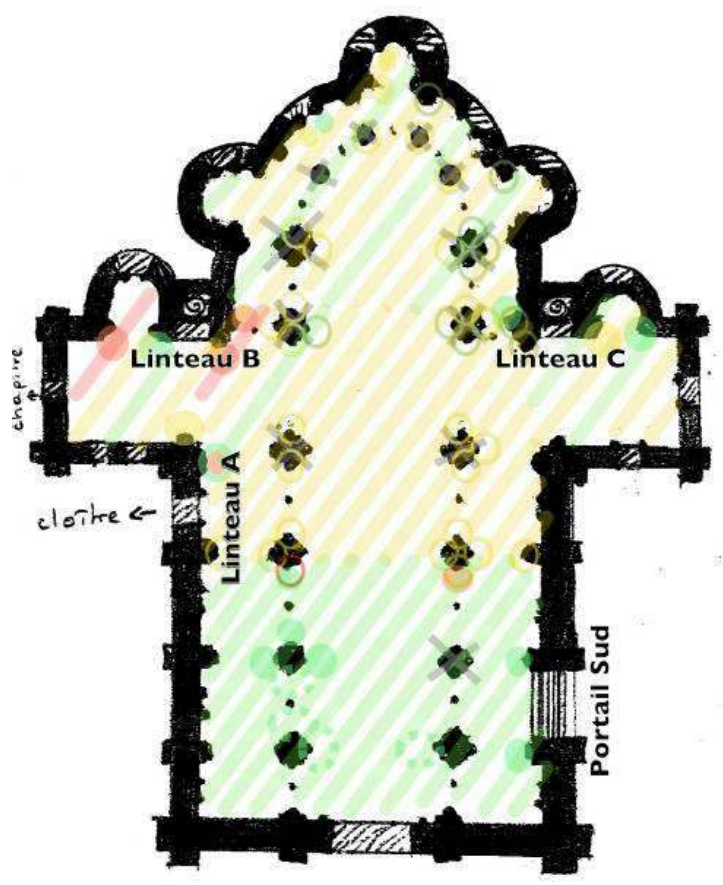

Image 6 : Organisation spatiale et thématique des sculptures.

Plan de l'église de Beaulieu-sur-Dordogne, coloré en fonction des éléments figurés par la sculpture. En vert : le végétal ; en jaune : des éléments animaux ; en rouge : des personnages humains. 